\title{
Information Flowing in Small-world network
}

\author{
Cui Wang
}

School of North China Electric Power University, Baoding 071000, China;

1543025473@qq.com

Keywords: Small-world Network, Analytic Hierarchy Process, Flow of information

\begin{abstract}
We build a small-world network model to evaluate the impact of information, regarding the participants as nodes, and taking the communication among participants as sides. We then propose the concept of importance degree of information and influence degree of participants to measure the ability of information diffusion and filter news. Combining with public opinion and interest, Analytic Hierarchy Process (AHP) is used to evaluate the importance degree of information. The flow of information is simulated in the small-word network, from which the speed of information diffusion can be figure out. We draw a conclusion that the importance of information and influence of participants are the main indexes to filter news from information.
\end{abstract}

\section{Introduction}

With the development of science and technology, social information system towards stronger. In the information age, social network has become the platform for human beings to create and share information, meanwhile the effects and influence of network is growing fast. In social network, any two participants are linked to each other if there is an interaction between them, through which new information can diffuse from one to another participant, eventually influence the whole network.

\section{Model overview and concepts definition}

From the perspective of information diffusion, we build the communication model based on the small-word network to reflect information dissemination. According to the requirements, our network should have the following functions:

- The network can clearly show how the information spreading between different participants, analyze the inherent value of information as well as measure the speed of information

- Based on the description of the problem, the index to filter which kind of information is news should be defined.

- We must pay more attention to the main factors that can effect the spreading of the information including: the value of the information itself, the importance of participants in the network, along with economic beckground of social network.

To better illustrate our Information Diffusion Model , the important elements and concepts based on small-world network are defined as follows:

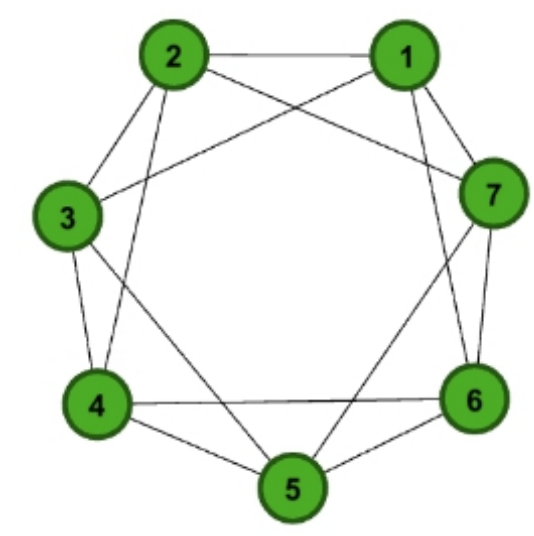

Figure 1.simple schematic of the small-world network 
Degree of node: the number of edges connected with the target node directly. In the Figure 1, the degree of each node is 2 .

Neighbor: the most close nodes to the target node. In the Figure1, node 7 and node 2 are neighbors to node 1 .

Second-degree node: the neighbors of the target node's neighbor. In the Figure 1, node 3 and node 6 are second-degree nodes of node1.

$\mathbf{N}$-degree node:the nodes in the whole network except the neighbors and second-degree node. In Figure 1 . node 4 and node 5 are $\mathrm{N}$-degree nodes of node 1.

Speed of information Diffusion: in the fixed network, speed is inversely proportional to time. We can obtain the time of information Diffusion in the whole network relative easily

Information source: the code who make the information.

\section{The importance degree of information}

In this part, the index system of the information comprehensive is evaluation is made, and the the comprehensive model is built by fuzzy comprehensive evaluation method. Then the value of information is applied and analyzed ${ }^{[1]}$.

The inherent value of the information is an essential factor in the dissemination of information. It is widely believed whether people spread a message based on his Interest in such topics. So, we make our minds to solve this problem by scoring based on AHP.

Our purpose is to measure the value of information. In our discussions, the value of information is equivalent to the degree of social concern. And the message must be grammatically correct, understandable and unambiguous as we assumed.

The AHP can be implemented in two simple consecutive steps in our model:

Computing the vector of criteria weights ${ }^{[2]}$.

Computing the matrix of option scores.

First, we must structure a set of evaluation criteria. We learn about topic of social concern from Internet[6]. At last, political, cultural, ecological, economic, others, the five listed topics are taken into account. By automatic indexer, we could get this wise comparison matrix A [7]

$$
A=\left(\begin{array}{ccccc}
1 & 3 & 5 & 1 & 5 \\
\frac{1}{3} & 1 & 2 & 4 & 2 \\
\frac{1}{5} & \frac{1}{2} & 1 & \frac{1}{5} & 1 \\
1 & \frac{1}{4} & 5 & 1 & 5 \\
\frac{1}{4} & \frac{1}{2} & 1 & \frac{1}{5} & 1
\end{array}\right)
$$

While subjective factors greatly affect the formation of the matrix, We always need to verify the consistency of the matrix A. The Consistency Proportional (CR) is described by the following formula

$$
C I=\frac{\lambda_{\max }-n}{n-1}, C R=\frac{C I}{R I}
$$

While subjective factors greatly affect the formation of the matrix, We always need to verify the consistency of the matrix $A$. The Consistency Proportional (CR) is described by the following formula

In this problem, $\mathrm{CR}=0.0228<0.100$.CR is small enough to prove that: $A$ is logical.And now, the vector of criteria weights can be shown here: 


$$
W=[0.36 \text { ( political), 0.24(cultural), 0.07(ecological), 0.26(economic), 0.07(others) }]
$$

The very message can contain up to two areas of information, so, we will get a exactly score for the message can be used in our model.

\section{The influence degree of participants}

the participant's influence in the network. It measures a node's ability to communicate with other nodes and to build the connection among different nodes in the whole network. To facilitate understanding and further analysis, we give the schematic diagram of the network model, shown in Figure 2.
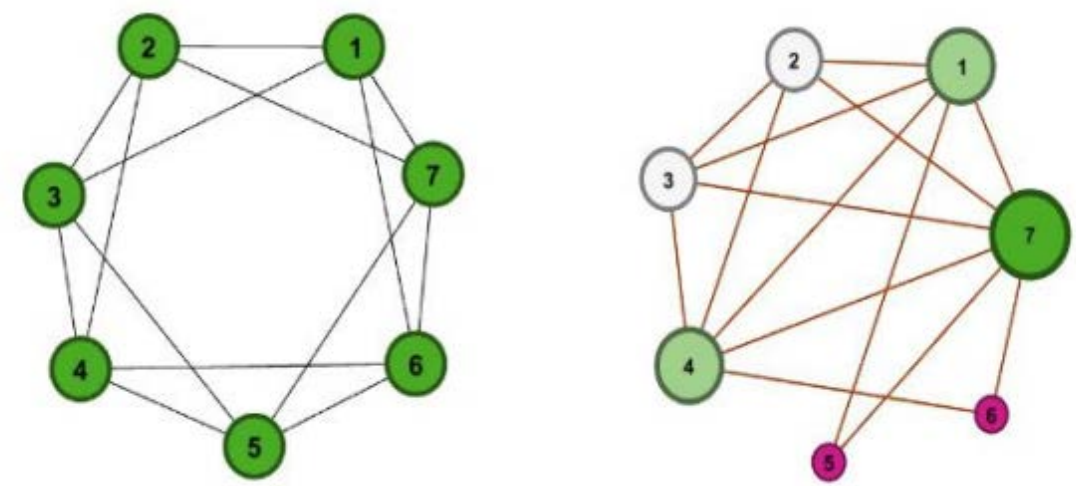

Figure 2. Before network reconnection ,the nodes with the same importance while after network reconnection, the nodes which connect more sides are more important

Assume that network $G=(V, E)$ is consists of $|V|=N$ nodes and $|E|=M$ sides. Node degree can be expressed as:

$$
k_{i}=\sum_{j \in G} \delta_{i j}
$$

If node i and node $j$ is not connected, $\delta_{i j}=0$.If node $i$ and node $j$ is connected, $\delta_{i j}=1$. As we can clearly see from the Figure 2, before the network reconnection, there are 7 codes and each code is connected with its neighbors and its two-degree node. After the network reconnection, the degree of each node has changed. The bigger of the degree, the greater size of node. From which we can conclude that the more edges the node has, the greater importance of the node in the network. meanwhile, there are no intensity differences in the network, so we regard it as unweighted network.

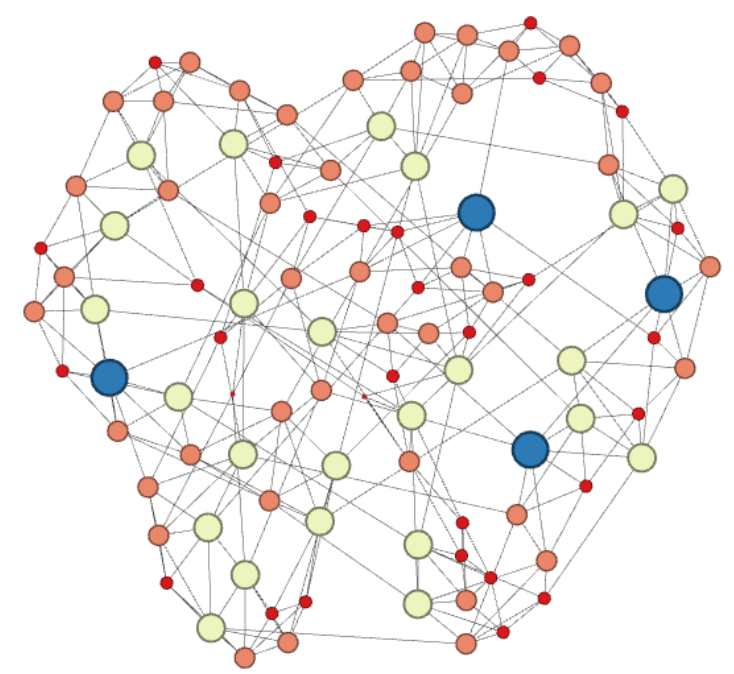

Based on the importance degree of information and influence degree of participants, we simulate the flow of information in a social network which has 100 codes. After a period of time, the 
dissemination of information in the network is shown as Figure 3. Based on the principle of small-world, the codes whole have greater degree are bigger in the small word.

\section{References}

[1] Liu S.L\&ets "Model of comprehensive Evaluation for the value of information"[J] (2007).Journal of Information Engineering University,8(1),118-119

[2] http : / / www. People - press. Org / 2012 / 09 / 27 /section-1 -watching - reading - and - listen ing -to -th e-news-3/ 